\title{
Acceleration schedules for a recirculating heavy-ion accelerator
}

\author{
W. M. Sharp and D. P. Grote \\ Lawrence Livermore National Laboratory, Livermore, California 94550
}

(Received 7 May 2002; published 18 September 2002)

\begin{abstract}
Recent advances in solid-state switches have made it feasible to design programmable, high-repetitionrate pulsers for induction accelerators. These switches could lower the cost of recirculating induction accelerators, such as the "small recirculator" at Lawrence Livermore National Laboratory (LLNL), by substantially reducing the number of induction modules. Numerical work is reported here to determine what effects the use of fewer pulsers at higher voltage would have on the beam quality of the LLNL small recirculator. Lattices with different numbers of pulsers are examined using the fluid/envelope code CIRCE, and several schedules for acceleration and compression are compared for each configuration. For selected schedules, the phase-space dynamics is also studied using the particle-in-cell code WARP3D.
\end{abstract}

DOI: 10.1103/PhysRevSTAB.5.094202

PACS numbers: 41.75.Ak, 41.85.Ja, 41.85.Ew

\section{INTRODUCTION}

A series of scaled experiments was carried out recently at the Lawrence Livermore National Laboratory (LLNL) to test the concept of a recirculating induction accelerator or "recirculator" $[1,2]$. The pulsed-power circuitry designed in 1995-1997 to drive the induction modules of this "small recirculator" attained the needed waveform control and repetition rate by using a parallel array of metal-oxidesemiconductor field-effect transistors (MOSFETs), which at the time had a voltage limit of about $500 \mathrm{~V}$. Because of this limit, the original small-recirculator design required an induction module in each available half-lattice period (HLP) of the ring in order to meet the goal of doubling the beam velocity during 15 laps. The pulsers for these modules constituted about half of the projected hardware cost of the small recirculator.

Advances in power-control devices since 1997 allow the design of solid-state induction-module pulsers with a substantially higher output voltage than those designed for the LLNL small recirculator. MOSFETs are now available with a maximum operating voltage of $1.2 \mathrm{kV}$, and insulated-gate bipolar transistors (IGBTs) can switch up to $3.3 \mathrm{kV}$, although the switching speed of these devices is about $200 \mathrm{~ns}$, compared with $20 \mathrm{~ns}$ for MOSFETs [3]. These devices can be connected in series or summed in an inductive adder [3] to obtain pulses of the desired voltage and waveform. Between 1997 and 1999, LLNL worked with First Point Scientific, Inc. (FPSI) to design and fabricate four prototype high-voltage pulsers for the small recirculator using the miniature inductive adder (MIA) developed by FPSI. The new pulsers delivered programmable positive or negative pulses up to $1 \mathrm{kV}$, and no obstacles were found to generating pulses up to $20 \mathrm{kV}$ for larger accelerators. This technology could lower the cost of the small recirculator by substantially reducing the number of acceleration modules, and it could simplify the development of pulsers for a driver-scale recirculator by avoiding the problem of connecting MOSFETs in series.
The possible use of higher-voltage pulsers raises the question of whether applying larger-amplitude but lessfrequent acceleration and control fields would seriously impair the beam quality of the LLNL small recirculator. This paper reports numerical work comparing lattices with between 5 and 34 pulsers, with several compression schedules being examined for each configuration. Although the parameters of the LLNL small recirculator are used here, we expect that the qualitative conclusions are also valid for the UMER electron recirculator being built at the University of Maryland [4].

\section{METHOD}

The numerical work to compare acceleration schedules has been done with the fast-running beam-dynamics code CIRCE [5]. The code combines an envelope description of the beam transverse dynamics with a fluidlike treatment of longitudinal dynamics. Although CIRCE assumes a constant beam emittance, it has previously been benchmarked against the three-dimensional (3D) particle-in-cell code WARP3D [6] and is found to model transverse and longitudinal dynamics acceptably in cases where emittance growth is unimportant. To facilitate the testing of acceleration and compression schedules with different numbers of pulsers, a subroutine has been added to CIRCE to set up the longitudinal electric potential $V(t)$ in each induction module. This two-step calculation first uses a modified version of an approach developed by Kim and Smith [7] to generate acceleration fields for self-similar compression in the absence of the longitudinal space-charge field. Longitudinal-control fields, called "ears" here, are then added to balance the longitudinal force due to the beam space charge.

As originally formulated, the Kim-Smith approach assumes that beam slices have ballistic trajectories between induction modules, referred to here as "cells." For the $i$ th slice, the velocity between the $n$th cell, centered at longitudinal position $s_{n}$, and the next one at $s_{n+1}$ is then 


$$
\beta_{n+1}^{i} c=\frac{s_{n+1}-s_{n}}{t_{n+1}^{i}-t_{n}^{i}} \equiv \frac{\delta s_{n}}{\delta t_{n}^{i}},
$$

where the slice arrival time $t_{n+1}^{i}$ is chosen so the beam current $I_{b}$ at $s_{n+1}$ is self-similar to that at $s_{n}$. Specifically, $I_{b}$ is a self-similar function of time $t$ and scales inversely with the beam duration $\Delta t$ provided that the $t^{i}$ values at each cell are chosen to give the same normalized beamframe times $\tau^{i} \equiv\left(t^{i}-t_{\text {mid }}\right) / \Delta t$, where $t_{\text {mid }}$ is the arrival time of the beam midpoint. Assuming that the beam is instantaneously accelerated at the midpoint of each cell, the voltage $V_{n}^{i}$ needed in the $n$th cell at time $t_{n}^{i}$ is then given approximately by

$$
V_{n}^{i} \approx \frac{\bar{\gamma}_{i}^{3} M c^{2}}{2 q e}\left[\left(\beta_{n+1}^{i}\right)^{2}-\left(\beta_{n}^{i}\right)^{2}\right]
$$

where $M$ and $q$ are the mass and charge state of beam ions, and $\bar{\gamma}_{i}$ is the Lorentz factor associated with $\bar{\beta}_{i}^{2} \equiv$ $\frac{1}{2}\left[\left(\beta_{n}^{i}\right)^{2}+\left(\beta_{n+1}^{i}\right)^{2}\right]$. This algorithm is sketched in Fig. 1. For a gap length of $L_{g}$, an analytic calculation shows that the assumption of instantaneous acceleration introduces a fractional error in $\delta t_{n}^{i}$ of about $\left[\left(\beta_{n+1}^{i}-\right.\right.$ $\left.\left.\beta_{n}^{i}\right) / 2 \bar{\beta}\right]^{2}\left(L_{g} / \delta s_{n}\right)$, which is less than $10^{-5}$ for all cases reported here.

The voltage estimate in Eq. (2) is suitable for a beam in a straight lattice, in which the design orbit coincides with the beam-pipe axis. In a circular accelerator like a recirculator, however, the head-to-tail velocity variation or "velocity tilt" needed for beam compression causes the lower-energy beam head to have a trajectory inside the design orbit, and the higher-energy tail has a trajectory outside it, provided that the bend fields do not vary over the pulse duration to counteract this tendency. This centroid displacement for a beam slice with a nonzero momentum error alters the path length of a slice in a bend and must be accounted for, Eq. (1). A simple calculation using the approximation of continuous focusing (often called the "smooth approximation") shows that, for electrostatic sector bends each with an occupancy $\eta_{b}$, a radius $\rho$, and a mean radius $\bar{\rho} \equiv \rho / \eta_{b}$, the beam displacement $X$ in the accelerator plane, averaged over the alternating-gradient (AG) flutter motion, is

$$
\bar{X} \approx \frac{1}{\frac{\sigma_{0}^{2}}{4 L^{2}}-\frac{K}{R^{2}}+\frac{2}{\rho \bar{\rho}}} \frac{2}{\bar{\rho}} \frac{\Delta p}{p} .
$$

Here, $R$ is the beam-pipe radius, $L$ is the half-lattice period, and $\sigma_{0}$ is the betatron phase advance over a full lattice period $2 L$ in the absence of space charge. The "momentum error" $\Delta p \equiv p-p_{0}$ is the difference between the local beam momentum $p \equiv \gamma \beta M c$ and the design momentum $p_{0}=\left[q e \gamma M E_{b x} \rho\right]^{1 / 2}$, which is the value for which an ion will stay on the design orbit in a sector bend with a radius $\rho$ and field strength $E_{b x}$. For a magnetic sector bend with a field strength $B_{b y}$, the design momentum becomes $p_{0}=q e \gamma M B_{b y} \rho$, but the $\bar{X}$ expression corresponding to Eq. (3) differs only in the factor $2 / \bar{\rho}$ being replaced by $1 / \bar{\rho}$. The phase-advance depression caused by the beam space charge is accounted for in Eq. (3) by the term proportional to the perveance $K$, given in SI units by

$$
K=\frac{1}{4 \pi \epsilon_{0}} \frac{2 q e I_{b}}{(\beta \gamma)^{3} M c^{3}} .
$$

For a sufficiently intense beam, this space-charge term causes the displacement of a beam with a linear velocity tilt to be "S shaped" rather than linear as a function of time. To lowest order in $\bar{X} / \rho$, the added path length due to bends can be accounted for in Eq. (1) by the substitution

$$
\delta s_{n} \rightarrow \delta s_{n}+\sum_{m=1}^{m_{b}} \frac{L_{b m}}{\rho_{m}} \bar{X}_{i}\left(s_{b m}\right),
$$

where $L_{b m}, \rho_{m}$, and $s_{b m}$ are, respectively, the length, the bend radius, and the axial location of $m_{b}$ bends between the induction cells. Since $\bar{X}_{i}$ depends on $\beta_{n+1}^{i}$ directly through $\Delta p / p$ and $\gamma_{i}$, and indirectly through $\sigma_{0}$ and the dependence of $I_{b}$ on $\Delta t$, Eq. (1) becomes a transcendental equation for $\beta_{n+1}^{i}$ and must be solved iteratively.

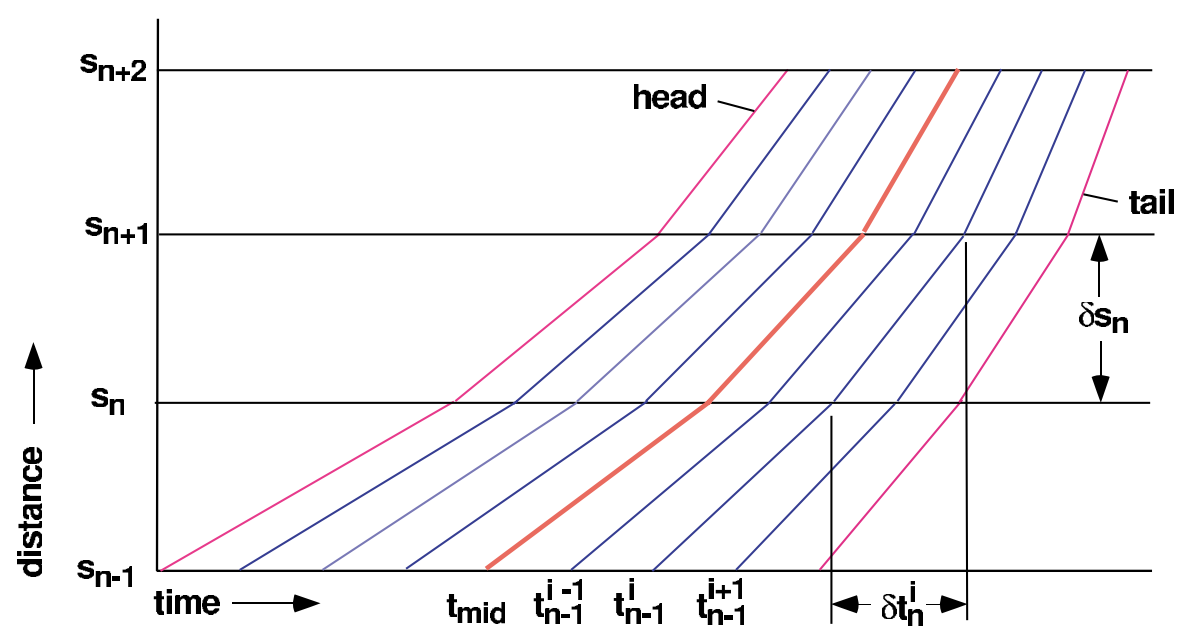

FIG. 1 (Color) Cartoon illustrating the basic algorithm for setting induction-cell voltages. Each beam slice has a ballistic trajectory between cell locations $s_{n}$, and for self-similar compression, the relative spacing of slices is kept constant at each cell. Cell voltages are then calculated as functions of time from the slope change of beam-slice trajectories. 
A straightforward procedure is used here to calculate the full waveforms for acceleration and longitudinal control. First, the scaled midpoint velocity $\beta_{\text {mid }}$ and the beam duration $\Delta t$ are specified as piecewise-continuous functions of longitudinal position $s$. The modified Kim-Smith algorithm is next used to generate appropriate waveforms for acceleration and compression only, ignoring the longitudinal component of the beam space-charge field. CIRCE is then run using these fields but with the longitudinal space-charge field artificially turned off, mimicking perfect longitudinal control. The beam current profile from this run is used to calculate the optimal control field in each acceleration gap. These fields are written along with the corresponding acceleration/compression fields to an external file and used in subsequent CIRCE runs. For selected cases, the same fields are also used in WARP3D simulations to corroborate the CIRCE results and to study emittance growth during acceleration.

\section{RESULTS}

A large number of CIRCE runs have been made to study the consequences of using higher-voltage pulsers in the LLNL small recirculator. The intention here is to highlight the effects of the number of cells and the rate of compression, not to choose an optimum acceleration schedule, so a somewhat simplified version of the small-recirculator lattice is used. The bore, axial length, and nominal field strengths of lattice elements match those of the experiment, but fringe fields are ignored, and sector bends are used instead of the more complicated "flat-plate" bends actually built. Also, to clarify the results, no errors in the strength or alignment of lattice elements are introduced, and dipole voltages are chosen to be matched to the beam velocity at the pulse midpoint. These idealizations should have little effect on the results presented here. Nominal parameters of the small recirculator are given in Table I, and a detailed description of the lattice is found in Ref. [8]. Using the nominal $500 \mathrm{~V}$ pulsers, induction cells are needed in 34 of the 40 half-lattice periods. Three HLPs are needed for a large-aperture section to insert the beam into the ring and to extract it, and another three-HLP section is planned for extraction halfway around the ring. Acceleration is not practical in these sections because of their size and complicated geometry.

For this nominal case with 34 cells, the specified four-to-one compression of the beam duration $\Delta t$ is obtained by imposing a velocity tilt as rapidly as possible, consistent with a maximum pulser voltage of $500 \mathrm{~V}$. The midpoint beam energy is taken to increase linearly with $s$ except in the insertion/extraction sections, where it is constant, and the beam-compression requirement is met by using a head-to-tail voltage increase $\Delta V=500 \mathrm{~V}$ in the first 13 cells on the first lap, followed by $\Delta V=0$ in the remaining cells. The first 13 waveforms that result are approximately triangular, with small deviations that
TABLE I. Nominal parameters of the LLNL small recirculator.

\begin{tabular}{lcc}
\hline \hline \multicolumn{3}{c}{ Beam parameters } \\
\hline Ion charge state & $q$ & 1 \\
Ion mass & $M$ & $39 \mathrm{amu}$ \\
Beam current & $I_{b}$ & $2 \rightarrow 8 \mathrm{~mA}$ \\
Kinetic energy & $\left(\gamma_{0}-1\right) M c^{2}$ & $80 \rightarrow 320 \mathrm{MeV}$ \\
Duration & $\Delta t$ & $4 \rightarrow 1 \mu \mathrm{s}$ \\
Rise/fall time & $\Delta t_{r}$ & $1 \rightarrow 0.25 \mu \mathrm{s}$ \\
\hline \multicolumn{3}{c}{} \\
\hline Circumference & Lattice parameters \\
Half period & $s_{\max }$ & $14.4 \mathrm{~m}$ \\
Pipe radius & $L$ & $36 \mathrm{~cm}$ \\
Number of laps & $R$ & $3.5 \mathrm{~cm}$ \\
\hline \hline
\end{tabular}

account for the transverse space-charge field, and the remaining ones are nearly flattopped. Hereafter, we refer to such pulses simply as "triangular" and "square." In addition, appropriate ear fields are added to the acceleration waveforms, as they are in all cases here, unless otherwise stated. Two views of the resulting dynamics are shown in Fig. 2. The time variation of the centroid displacement $X$ at the end of the last lap is shown in Fig. 2a, while Fig. 2b shows $X$ at the beam head during the final lap. The two types of plots are referred to hereafter, respectively, as "snapshots" and "histories."

The head-to-tail slope in $X$ evident in Fig. 2a results from the $1.2 \%$ velocity tilt that remains at the end of 15 laps. For the beam and lattice parameters used here, Eq. (3) predicts the average displacement of the beam ends to be $\bar{X} \approx \pm 0.255 \mathrm{~cm}$, which is in fair agreement with the final snapshot. It is clear from Fig. $2 b$, however, that the beam head is undergoing substantial betatron oscillation after the 15 laps, and a history of the beam tail shows a similar amplitude of oscillation, but with a positive average displacement. This betatron motion, which is seen to some extent in all cases in this paper, arises because each pulse with a nonzero $\Delta V$ causes an abrupt change in the velocity tilt $\Delta p / p$ without significantly changing $X$. Since a change in $\Delta p / p$ is seen from Eq. (3) to alter the "equilibrium" displacement $\bar{X}$, the centroid is mismatched by an amount $\Delta X \sim \delta(\Delta p / p)$, and a simple calculation using Eq. (2) shows that

$$
\delta\left(\frac{\Delta p}{p}\right) \approx \frac{q e \Delta V}{2 \bar{\gamma}_{\mathrm{mid}} \bar{\beta}_{\mathrm{mid}}^{2} M c^{2}},
$$

where $\bar{\beta}_{\text {mid }}$ is the average of $\beta_{\text {mid }}$ before and after the cell and $\bar{\gamma}_{\text {mid }}$ is the corresponding Lorentz factor. The mismatches introduced in successive cells should add in a Markovian sense because the betatron wavelength of centroid motion is typically uncorrelated with the cell spacing. Therefore, if $N$ pulses are used to give a specified energy increase and velocity tilt, so that $\Delta V \sim N^{-1}$, we expect the accumulated betatron amplitude to have the approximate scaling 



FIG. 2. (Color) Beam-centroid displacement for the nominal lattice with 34 induction cells per lap, illustrated by (a) a "snapshot" showing the displacement along the pulse at the end of 15 laps and (b) a "history" of the beam head during the last lap.

$$
\delta X \sim N^{1 / 2} \Delta V \sim N^{-1 / 2} .
$$

Since the frequency of betatron oscillation for a beam slice depends on the local perveance, betatron motion initiated near the ends by this mechanism will eventually get out of phase with the motion of the higher-density beam midsection, leading to the ripples seen in Fig. $2 \mathrm{a}$ near the beam ends.

\section{A. Effects of pulser number}

Several effects of using fewer pulsers at a higher voltage are illustrated here by comparing the nominal case with 34 cells to cases with $5,8,10$, and 18 cells per lap. These particular layouts have been chosen because, except for the case with 18 pulsers, they allow acceleration modules to be spaced equally around the ring, facilitating the setting of dipole voltages. As in the nominal case, equal cell spacing is not feasible for the 18-pulser case because of the two sections for insertion and extraction. For each layout, $\beta_{\text {mid }}$ and $\Delta t$ are specified by the same functions as in the case with 34 cells, so the midpulse voltage scales inversely as the number of cells per lap, and the number of triangular pulses changes proportionally. However, due to the larger cell spacing, the transition from triangular to square pulses does not in general coincide with a cell location, so the final waveform with nonzero $\Delta V$ generated by the algorithm here is approximately trapezoidal, again with small deviations due to space charge.

The lattices with 18 and 10 cells per lap, which use triangular or trapezoidal pulses, respectively, in the first seven and four cells, both show acceptably smooth compression. Final-lap histories of the beam-head displacement for these two cases are shown in Fig. 3. Comparing these with Fig. 2b, it is apparent that betatron motion becomes substantially worse going from 34 to 18 cells and remains nonperiodic, but it is seen to have reduced amplitude for the ten-cell case and to be more nearly periodic. This improvement seen in going from 18 to 10 cells results from the uniform cell spacing possible with the smaller number of cells. When induction cells are added to the insertion/extraction sections of the lattices with 18 and 34 cells, making periodic lattices with, respectively, 20 and 40 cells, the betatron motion at the beam ends becomes very similar to that for the ten-cell case. We note that
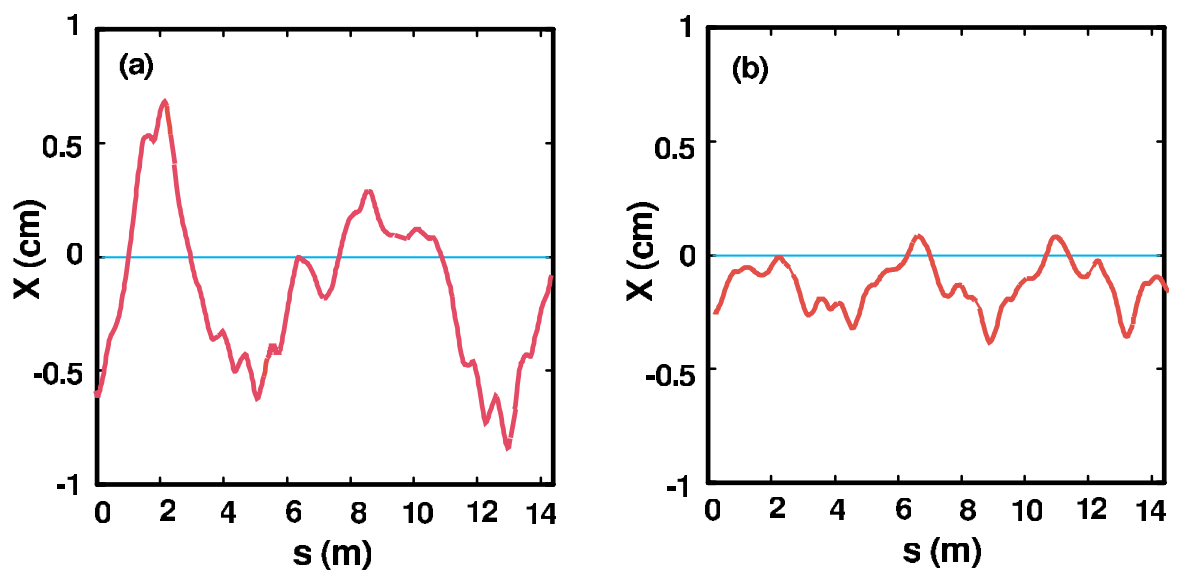

FIG. 3. (Color) Final-lap "histories" of beam-centroid displacements using (a) 18 cells per lap and (b) 10 cells per lap. Here, a velocity tilt is imposed in the first seven and first four cells, respectively, giving the nominal four-to-one reduction in beam duration. 
final amplitude of betatron oscillations seen in the cases with 10,20 , and 40 cells per lap are roughly in agreement with Eq. (7), although the small values of $N$ in these cases make this scaling dubious. However, the simple model used to obtain the scaling misses the enhancement of $\delta X$ caused by nonuniform cell spacing, so the betatron motion seen in the cases with 18 and 34 cells exceeds the analytic estimate.

The enhanced betatron amplitude resulting from nonuniform cell spacing is illustrated in Fig. 4. Here, centroid histories in a lattice with ten uniformly spaced cells are compared with those in a similar lattice in which the first and sixth cells have been shifted clockwise by a halflattice period, leaving the other cells fixed. When the longitudinal component of the beam space-charge field is artificially turned off and no longitudinal-control fields or ears are applied, the final betatron amplitude is found to be roughly a factor of 3 higher in the nonuniform lattice, as seen by comparing Fig. $4 \mathrm{a}$ with Fig. $4 \mathrm{~b}$. In both cases, the betatron motion is nearly sinusoidal because the mismatch is applied in the first lap by four successive triangu- lar pulses. Since these cells span approximately a single betatron wavelength, the mismatch is, in effect, an initial perturbation on a slice trajectory, and the oscillation persists for the remainder of the run because there is no phase-mix damping in this envelope calculation. Including the space charge and ears is seen in Figs. $4 \mathrm{c}$ and $4 \mathrm{~d}$ to substantially increase the amplitude of betatron motion and make it nonperiodic. The amplitude is larger because ear fields in these ten-cell cases become substantially larger than the nominal acceleration voltage as the beam compresses. The nonperiodic motion results from the application of ear fields at times that are uncorrelated with the betatron phase, giving the beam ends effectively random transverse kicks several times during each betatron period. Because of this nonperiodic betatron motion, it is difficult to quantify the increase in amplitude caused by the nonuniform cell spacing. However, if the curves are smoothed to remove the AG flutter motion, the difference between the maximum and minimum transverse excursions seen in Fig. $4 \mathrm{~d}$ is roughly a factor of 2 larger than that for the case of Fig. $4 \mathrm{c}$ with uniform cell spacing. To give the correct

\section{space charge off}
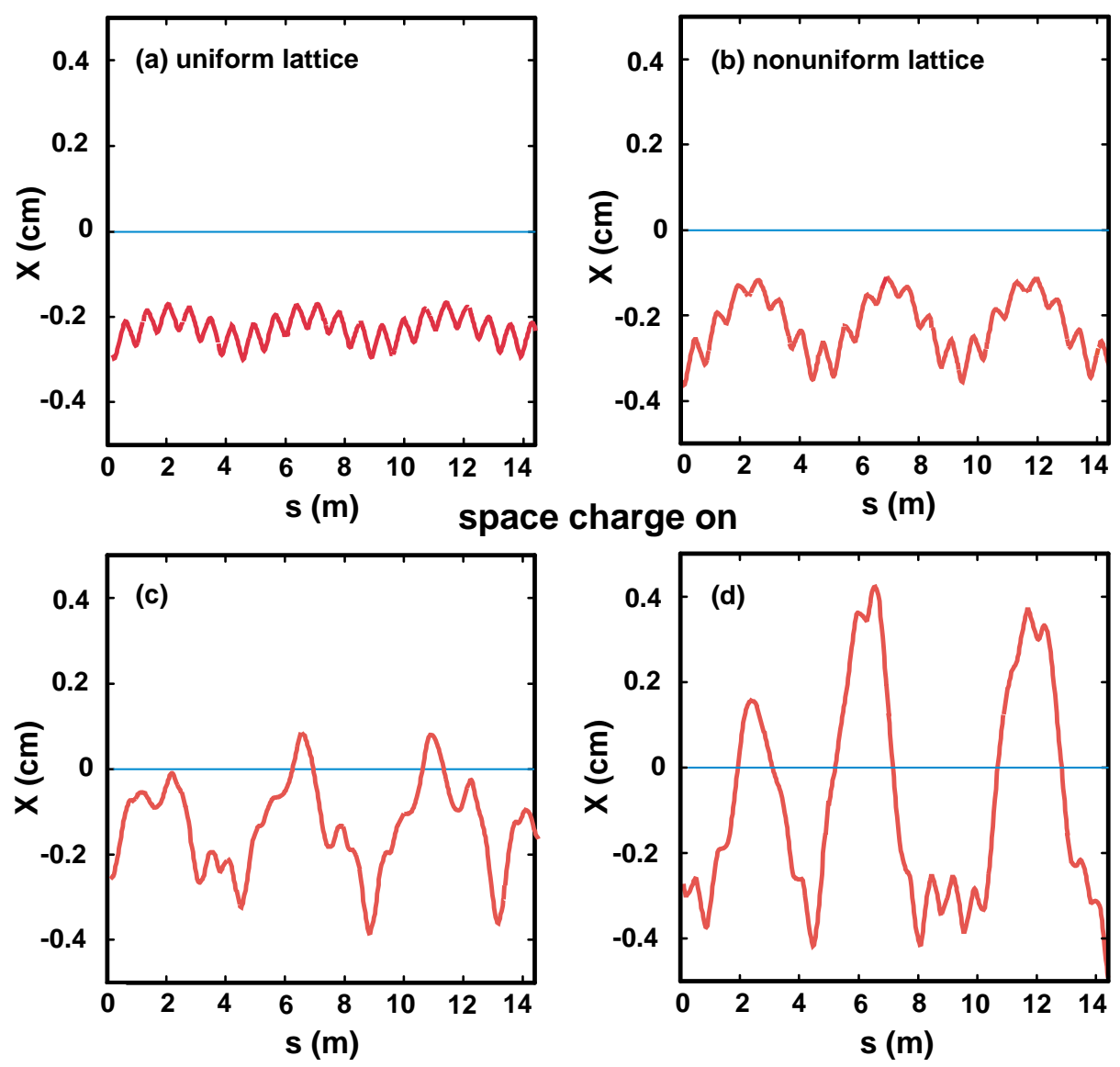

FIG. 4. (Color) Final-lap centroid histories showing the effects of nonuniform acceleration. Cases with the longitudinal space-charge field and the "ear" fields artificially turned off are shown (a) for a lattice with ten uniformly spaced cells and (b) for a similar lattice with the first and sixth cells moved clockwise by one half-lattice period. The corresponding cases including longitudinal space charge and ears are shown, respectively, in (c) and (d). Note that (c) shows the same data as Fig. 3b, but the vertical scale is changed to match the other plots here. 
average longitudinal-control force, the ear fields here scale inversely with the distance between cells, so when cells have nonuniform spacing, the ear amplitudes show a similar variation, contributing to the higher betatron amplitude seen in Fig. 4d.

In runs using five or eight cells per lap, the beam-end betatron oscillations become large enough to invalidate the numerical model in CIRCE. Apart from the fact that the acceleration voltage for these cases exceeds the design limit of the FPSI pulsers being developed for the small recirculator, these large oscillations prohibit the use of triangular waveforms when there are eight or fewer cells.

\section{B. Effects of pulser waveforms}

The beam-end mismatch introduced by head-to-tail voltage variation in waveforms can be reduced by using a smaller $\Delta V$ in a correspondingly larger number of induction cells. For a given lattice and overall compression, a progressive reduction in betatron motion at the beam ends is seen when a schedule with triangular pulses is replaced by one using a larger number of trapezoidal pulses. This improvement is illustrated by the final-lap centroid histories in Fig. 5, using trapezoidal pulses, respectively, for the first 10, 50, 100, and 150 waveforms. Each case here uses the same ten-cell lattice, and the head-to-tail voltage increase $\Delta V$ on the trapezoidal pulses has been chosen to give the same four-to-one final reduction of the beam duration. The case in Fig. 5a, with ten trapezoidal pulses, is only slightly better than that with seven triangular pulses, shown in Fig. 3b, but a major decrease in betatron motion is evident in Fig. $5 \mathrm{~b}$ going from 10 to 50 trapezoidal pulses. At the same time, the previously noted nonperiodicity due to ear fields decreases in proportion with the betatron motion. Some further improvement is also seen in Figs. 5c and 5d, where progressively smaller voltage tilts are needed. From these cases and others with uniform cell spacing, it appears that betatron motion introduced at the beam ends by a voltage tilt becomes negligible when momentum tilt $\Delta p / p$ changes in a cell by less than about $0.1 \%$. Using Eq. (6), this criterion can be written to lowest order in $\bar{\beta}_{\text {mid }}^{2}$ as

$$
\frac{q e \Delta V}{\frac{1}{2} \bar{\beta}_{\mathrm{mid}}^{2} M c^{2}} \leq 0.004
$$

where $\frac{1}{2} \bar{\beta}_{\text {mid }}^{2} M c^{2}$ is the beam kinetic energy in the nonrelativistic limit. The cases in Fig. 5 with 50 or more
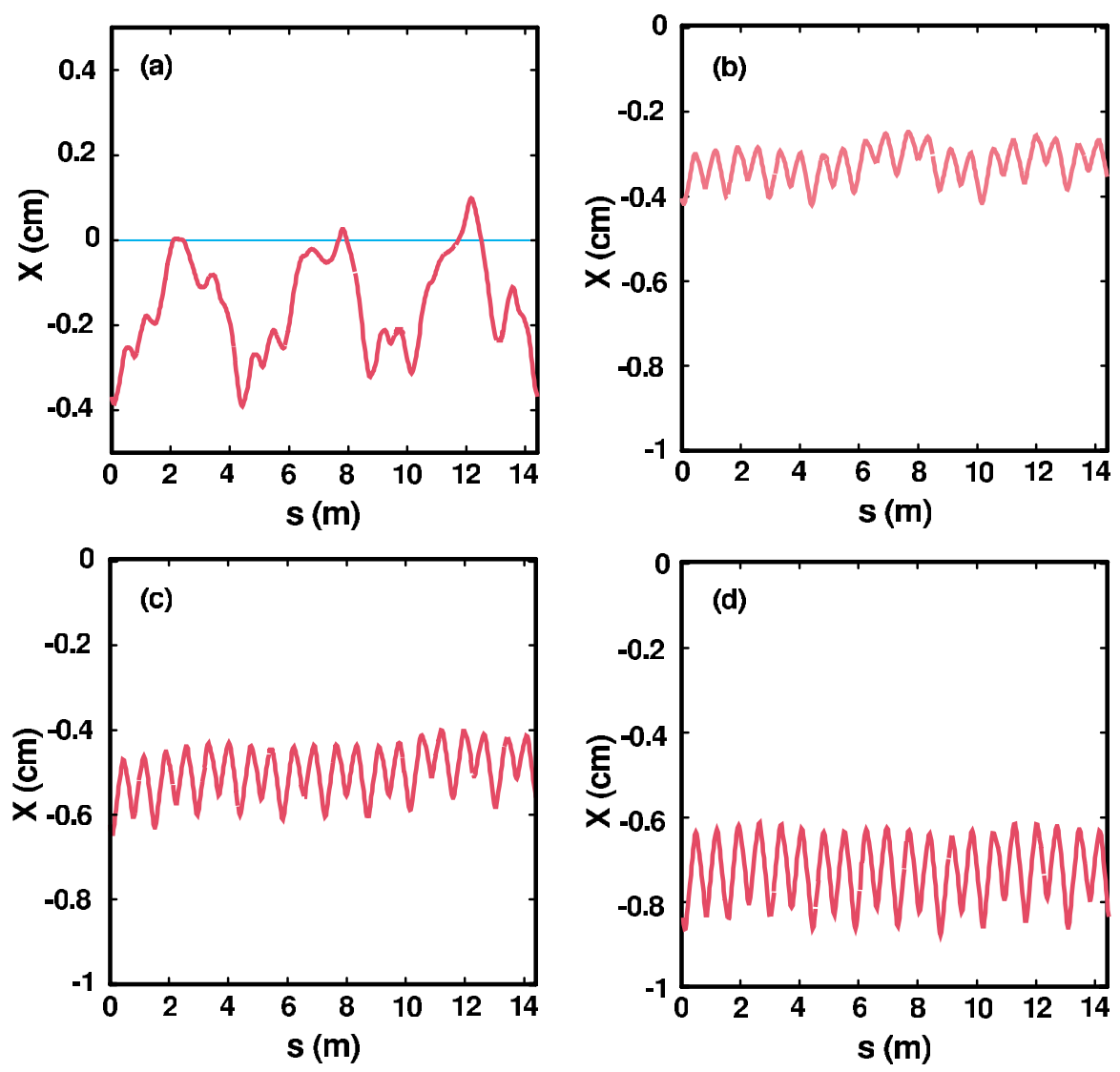

FIG. 5. (Color) Final-lap centroid histories showing the effects of applying a velocity tilt more gradually in a lattice with ten equally spaced cells. Trapezoidal pulses have been applied in the first (a) 10, (b) 50, (c) 100, and (d) 150 cells, with the head-to-tail voltage increase adjusted to give the same overall compression. Note that a different vertical scale is used in (a) to accommodate the larger centroid swing. 

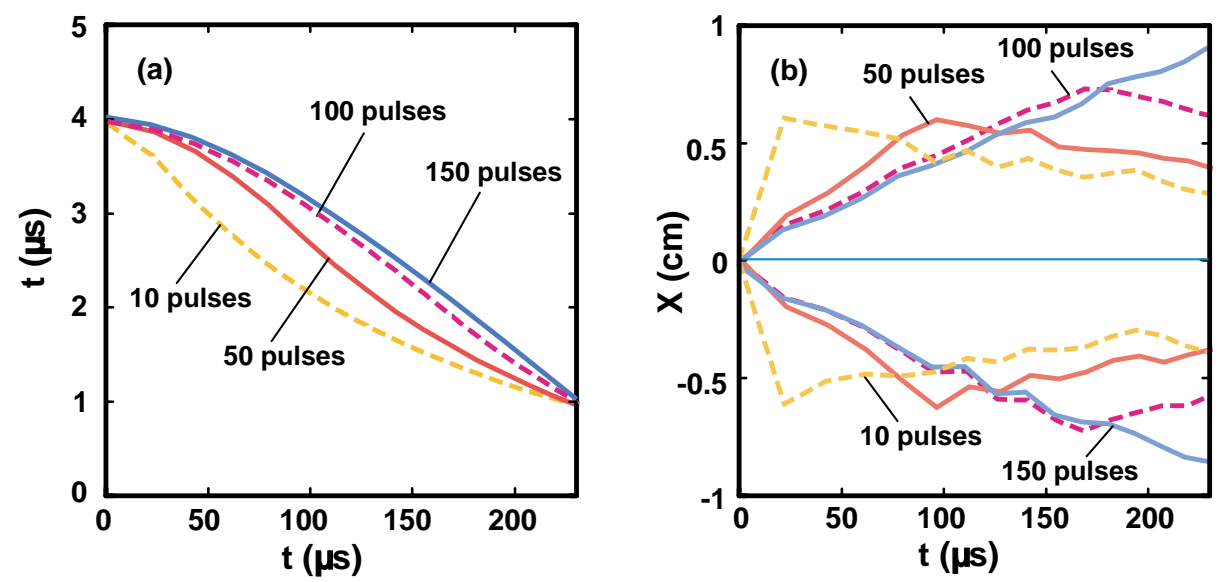

FIG. 6. (Color) (a) Beam duration and (b) the head and tail centroid displacement over 15 laps for compression schedules using ten cells per lap and trapezoidal waveforms for the first 10, 50, 100, and 150 pulses. The voltage variation in the trapezoidal pulses has been adjusted to give the same final compression.

trapezoidal pulses satisfy this criterion, but the case with ten trapezoidal pulses violates it.

Another feature seen in Fig. 5 is an increased average displacement as more trapezoidal pulses are used. Because such pulses introduce a velocity tilt more gradually than triangular ones, the beam duration decreases more
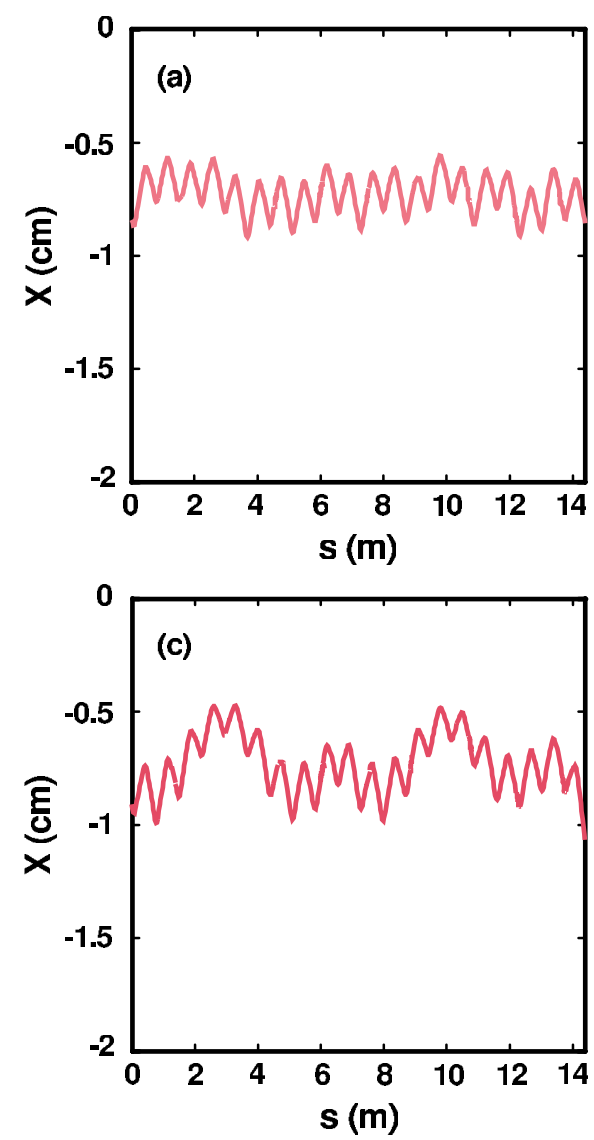

slowly at first, and the beam length actually increases due to the increasing velocity. Beam durations for the ten-cell cases with 10,50,100, and 150 trapezoidal pulses are compared in Fig. 6a. As the number of trapezoidal pulses increases and the voltage tilt is reduced, $\Delta p / p$ reaches its maximum of about $3 \%$ later in the acceleration sequence.
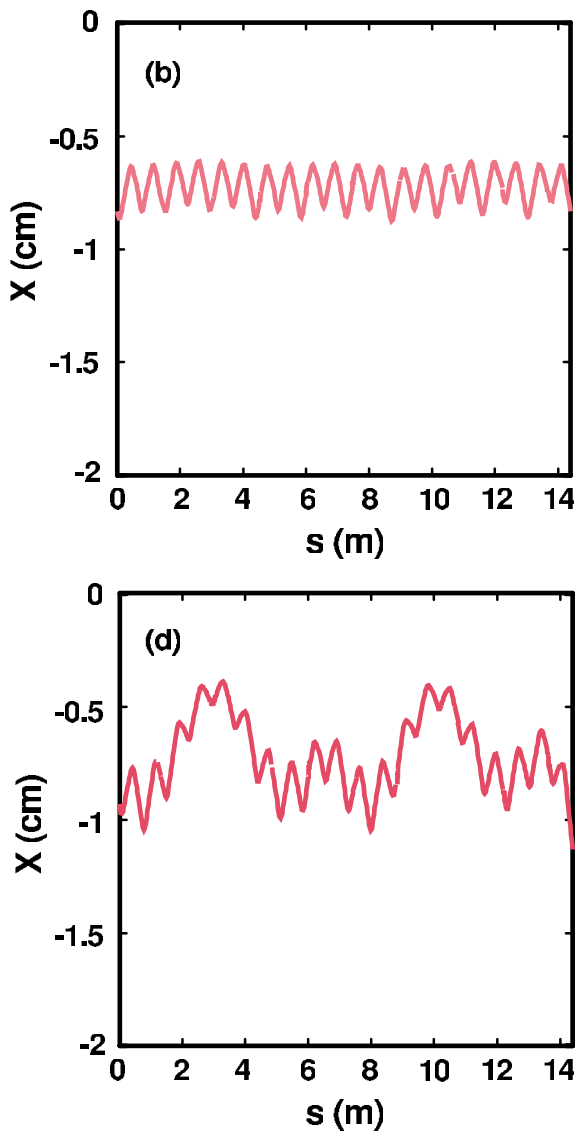

FIG. 7. (Color) Final-lap centroid histories showing the effects of applying a velocity tilt gradually in lattices with (a) 8, (b) 10, (c) 18, and (d) 34 cells per lap. Here, trapezoidal pulses are used for all waveforms, with the head-to-tail voltage increase adjusted to give the same overall compression. 
Consequently, the maximum average displacement estimated from Eq. (3) increases with the number of trapezoidal pulses in these cases due to the approximate $\beta^{-1}$ scaling of $\sigma_{0}$ found for magnetic focusing. This increase is evident in the plots of head and tail displacement in Fig. 6b. Since the sensitivity to lattice and field errors is expected to be similar for all these cases, choosing an optimum acceleration schedule for a recirculator entails balancing this increased displacement of the ends against the reduced betatron motion found with a more gradual introduction of velocity tilt. It follows that the velocity tilt should be introduced as rapidly as possible without initiating betatron oscillations at the beam ends, as determined by the criterion of Eq. (8).

As in the ten-cell case, the other lattices examined show some reduction in betatron amplitude when the velocity tilt is applied more gradually, as seen in Fig. 7. Here, final-lap histories are shown for four cases ranging from 8 to 34 cells per lap, each with trapezoidal pulses used for all waveforms and $\Delta V$ adjusted to give the same overall compression. The ten-cell case in Fig. $7 \mathrm{~b}$ clearly shows the least betatron motion. Slightly more is seen in the eight-cell case of Fig. 7a, and another lattice with five cells per lap had roughly twice the betatron amplitude in the final lap compared with the eight-cell case. The two cases in Fig. 7 with more than ten cells per lap show a substantial betatron amplitude due to the nonuniform spacing of cells, even though all cases shown satisfy the criterion in Eq. (8) for negligible betatron motion due to voltage tilt. This result suggests that nonuniform cell spacing acts as an independent source of mismatch.

Besides reducing the mismatch at the beam ends, the use of trapezoidal acceleration pulses rather than triangular ones makes more efficient use of the induction cores. The volt seconds required for a trapezoidal pulse are nearly the same as for a flat pulse, whereas a triangular pulse uses only about half that value. An added advantage of trapezoidal pulses is that the increase of the midpoint beam energy can remain the same on each lap, so to a good approximation, the required dipole voltage increases quadratically with time during the 15 laps, and if the cells are uniformly spaced, the same voltage waveform can be applied to every dipole.

\section{Emittance growth}

Several of the acceleration schedules discussed here have been tested by the 3D particle-in-cell code WARP3D [6] using the acceleration and ear fields generated by CIRCE. Although WARP3D is able to model the fringe fields and higher-order multipoles of lattice elements accurately, the same hard-edged, linearized elements assumed in CIRCE were used in these tests in order to highlight the effects of the schedules on the beam phase-space distribution, particularly the emittance. Since the beam density in WARP3D runs is taken to be initially uniform, CIRCE was rerun in

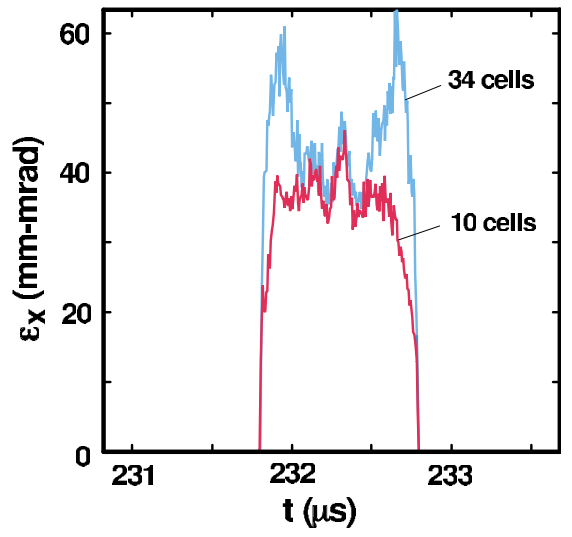

FIG. 8. (Color) Snapshots of the $x$ emittance after 15 laps for a lattice with 34 cells per lap and one with 10. Trapezoidal pulses adjusted to give the same overall compression have been used in all cells.

these cases with that model, so that the two codes would have similar beam-end dynamics, and magnetic sector bends were specified in both codes due to small differences in the modeling of electric sector bends. Also, trapezoidal waveforms were used for all acceleration pulses to minimize betatron motion.

As expected from earlier benchmark tests, the two codes predict similar longitudinal dynamics for the recirculator beam. In the cases compared, WARP3D gives virtually the same compression and velocity tilt as CIRCE, although details of the betatron motion of slices differ, and WARP3D snapshots of the beam current show longwavelength waves with an amplitude of about $5 \%$ of the peak current, suggesting small errors in the ear fields or their timing. Comparing WARP3D runs using 10 and 34 cells per lap, we find, as in CIRCE runs, that the final betatron amplitude is substantially higher for the case with 34 cells. However, the amplitude is noticeably higher in both cases than in the corresponding CIRCE runs, due mainly to the WARP3D beam being initialized with a nonequilibrium distribution. The particle simulations with 34 cells also show a larger and denser "halo" of unconfined particles near the ends than the ten-cell simulations, resulting in the much higher $x$ emittance near the ends seen in Fig. 8. For both cases, the normalized emittance near the midpoint increases about $68 \%$ during the 15 laps, again due mainly to the nonequilibrium initialization, but the increase is greater than $165 \%$ at two maxima near the ends when 34 cells are used. In contrast, no emittance growth above the midpoint value is seen in the ten-cell case. This enhanced emittance growth near the beam ends for 34 cells appears to be another effect of nonuniform cell spacing, since it is not seen in WARP3D runs in lattices with 20 or 40 cells.

\section{CONCLUSIONS}

From CIRCE and WARP3D simulations of the LLNL small recirculator, it appears that the beam can be accelerated and compressed using as few as five induction cells per 
lap, provided that the velocity tilt is introduced gradually enough to avoid the initiation of betatron oscillations near the beam ends. Triangular waveforms can be used when there are ten or more cells, but using a larger number of trapezoidal pulses to produce the velocity tilt reduces the amplitude of betatron oscillations. A layout having ten cells placed uniformly around the ring, with trapezoidal pulses used on perhaps the first five of the 15 laps, appears optimum for the small recirculator. In this case, the acceleration waveform has a nominal voltage of about $1.5 \mathrm{kV}$ and needs a head-to-tail voltage tilt of about $11.5 \%$. This schedule produces reasonably small betatron oscillations at the beam ends in the absence of lattice errors, while still keeping the beam centroid displacement less than $0.7 \mathrm{~cm}$. When fewer than ten cells are used, acceptable acceleration schedules can be worked out using a sufficient number of trapezoidal pulses, but the amplitude of betatron motion is higher than the ten-cell case, and the nominal voltage is larger than can be generated with the MIA pulsers developed for the small recirculator.

The main advantage of using MIA pulsers is the expected cost reduction due to the smaller number of pulsers, and there are further small savings due to such things as smaller housings for the pulsers and simpler mechanical alignment. These savings are partly offset by the need for more induction-core material. A second advantage of using MIA pulsers compared with a parallel-array MOSFET or IGBT architecture is their ability to generate bipolar pulses. It is found in the cases discussed here that ear voltages can exceed the acceleration voltage as the beam approaches full compression, which would make longitudinal control difficult with unipolar pulsers. The required ear voltages could, of course, be reduced by lengthening the rise and fall times of the beam current, thereby reducing the longitudinal space-charge field. However, allowing bipolar pulses increases the flexibility of the system.

A substantial amount of additional work is needed to complete this preliminary study. As mentioned, the lattice used here was somewhat idealized. To calculate usable acceleration fields, the lattice elements in the code should include realistic fringe fields, and flat-plate dipoles should be used instead of the sector bends used here. Coding should be added to calculate the volt seconds required in the in- duction cores in order to determine whether the calculated waveforms can be generated with the existing cores. Finally, a wider range of compression schedules should be examined in order to find one that simultaneously minimizes the centroid displacement at the beam ends and the final velocity tilt.

\section{ACKNOWLEDGMENTS}

This work was performed under the auspices of the U.S. Department of Energy by the University of California Lawrence Livermore National Laboratory under Contract No. W-7405-ENG-48.

[1] J. J. Barnard, F. Deadrick, A. Friedman, D. P. Grote, L. V. Griffith, H. C. Kirbie, V. K. Neil, M. A. Newton, A. C. Paul, W. M. Sharp, H. D. Shay, R. O. Bangerter, A. Faltens, C. G. Fong, D. L. Judd, E. P. Lee, L. L. Reginato, S. S. Yu, and T. F. Godlove, Phys. Fluids B 5, 2698-2706 (1993).

[2] L. Ahle, T. C. Sangster, J. Barnard, C. Butkhart, G. Craig, A. Debeling, A. Friedman, W. Fritz, D. P. Grote, E. Halaxa, R. L. Hanks, M. Hernandez, H.C. Kirbie, B. G. Logan, S. M. Lund, G. Mant, A. W. Molvik, W. M. Sharp, and C. Williams, Nucl. Instrum. Methods Phys. Res., Sect. A 464, 557-562 (2001).

[3] E. G. Cook, in Proceedings of the 20th International Linac Conference, Monterey, CA, 2000, edited by A. Chao, eConf C000821, We103, 2000.

[4] P. G. O'Shea, M. Reiser, R. A. Kishek, S. Bernal, H. Li, M. Pruessner, V. Yun, Y. Cui, W. Zhang, Y. Zou, T. Godlove, D. Kehne, P. Haldemann, and I. Haber, Nucl. Instrum. Methods Phys. Res., Sect. A 464, 646-652 (2001).

[5] W. M. Sharp, J. J. Barnard, D. P. Grote, S. M. Lund, and S. S. Yu, in Computational Accelerator Physics, edited by Robert Ryne, AIP Conf. Proc. No. 297 (AIP, New York, 1994), pp. 540-548.

[6] D. P. Grote, A. Friedman, I. Haber, and S. S. Yu, Fusion Eng. Des. 32-33, 193-200 (1996).

[7] C. H. Kim and L. Smith, Lawrence Berkeley Laboratory Report No. LBL-19137, 1985.

[8] J. J. Barnard, M. D. Cable, D. A. Callahan, T. J. Fessenden, A. Friedman, D. P. Grote, D. L. Judd, S. M. Lund, M. A. Newton, W. M. Sharp, and S. S. Yu, Fusion Eng. Des. 32-33, 247-258 (1996). 